\title{
MAXIMUM AND MINIMUM PROPERTIES OF THE TEMPERATURE IN LINEAR THERMOELASTICITY*
}

BY

W. A. DAY

Hertford College, Oxford

Introduction. We study the thermoelastic equations

$$
\begin{aligned}
& \frac{\partial^{2} \theta}{\partial x^{2}}=\frac{\partial \theta}{\partial t}+a \frac{\partial^{2} u}{\partial x \partial t} . \\
& \frac{\partial^{2} u}{\partial x^{2}}=a \frac{\partial \theta}{\partial x}+b \frac{\partial^{2} u}{\partial t^{2}} .
\end{aligned}
$$

The derivation of these equations from the standard equations of homogeneous and isotropic linear thermoelasticity, as presented by Carlson [1], is to be found in [2] and will not be repeated here. We remark, though, that $\theta(x, t)$ is the temperature, and $u(x, t)$ is the displacement in a slab $0 \leqslant x \leqslant 1$ which has unit (scaled) thickness. The coupling constant $a^{2}$, denoted by $a$ in [2], measures the interaction between thermal and mechanical effects, and the constant $b$ measures the effect of inertia.

Equation (2) is just the equation of motion

$$
\partial \sigma / \partial x=b \partial^{2} u / \partial t^{2}
$$

in which

$$
\sigma=\partial u / \partial x-a \theta
$$

is the stress.

The quasi-static theory is obtained by setting $b=0$, thereby ignoring inertia altogether; if we were to adopt that theory we should conclude that the stress is independent of the coordinate $x$, that is to say

$$
\sigma(x, t)=\sigma(y, t) \quad(0 \leqslant x, y \leqslant 1) .
$$

The thermoelastic theory generalizes the classical theory of heat conduction, according to which the slab is rigid and the temperature satisfies the heat equation

$$
\partial^{2} \theta / \partial x^{2}=\partial \theta / \partial t
$$

Received November 15, 1983. 
Let us consider a continuous function $\varphi(x, t)$, defined in the half-strip $S\left(t_{0}\right)=[0,1]$ $\times\left[t_{0}, \infty\right)$ of the $x, t$-plane. If $R \subset S\left(t_{0}\right)$ is any closed rectangle of the form $R=[0,1] \times$ $\left[t_{1}, t_{2}\right]$ we denote by $d R$ the parabolic boundary of $R$, that is to say that part of the boundary of $R$ which is formed from the three line segments

$$
[0,1] \times\left\{t_{1}\right\}, \quad\{0\} \times\left[t_{1}, t_{2}\right], \quad\{1\} \times\left[t_{1}, t_{2}\right],
$$

which comprise the base and the two vertical sides of $R$.

We shall say that $\varphi$ has the maximum property in $S\left(t_{0}\right)$ if $\max _{R} \varphi=\max _{d R} \varphi$, and that $\varphi$ has the minimum property in $S\left(t_{0}\right)$ if $\min _{R} \varphi=\min _{d R} \varphi$, for every such $R \subset S\left(t_{0}\right)$.

As is very well known: If $\varphi$ is continuous in $S\left(t_{0}\right)$, and if $\partial^{2} \varphi / \partial x^{2}$ and $\partial \varphi / \partial t$ exist and are continuous in the interior of $S\left(t_{0}\right)$, then $\varphi$ has the maximum property in $S\left(t_{0}\right)$ provided that $\partial^{2} \varphi / \partial x^{2} \geqslant \partial \varphi / \partial t$, and $\varphi$ has the minimum property in $S\left(t_{0}\right)$ provided that $\partial^{2} \varphi / \partial x^{2} \leqslant$ $\partial \varphi / \partial t$. If $\varphi$ is a solution of the heat equation it has both the maximum property and the minimum property in $S\left(t_{0}\right)$.

We propose to study solutions $\theta, u$ of the thermoelastic equations which are defined in the half-strip $S(0)=[0,1] \times[0, \infty)$ and to ask whether it is still the case that, as the classical theory predicts, $\theta$ has the maximum and minimum properties in $S(0)$.

So wide a statement is simply false for the thermoelastic equations. Nonetheless we shall prove, what is our main result, that $\theta$ has the maximum property if there is a net flow of heat out of the slab at each instant, and that $\theta$ has the minimum property if there is a net flow into the slab at each instant, so long as we confine our attention to a half-strip $S\left(t_{0}\right) \subset S(0)$ where $t_{0} \geqslant 0$ is some finite number, chosen appropriately. In effect, we may say that, if the net heat flow is of constant $\operatorname{sign}, \theta$ will have either the maximum property or the minimum property once the 'noise' generated by the initial data on $t=0$ has had time to die down. This result is different from the results of the articles $[3,4]$ but it reinforces the same general point that, if a proposition which holds for the heat equation extends at all to thermoelasticity, it is most likely to be valid after a sufficient lapse of time.

1. Maximum and minimum properties of the temperature. We suppose always that $a>0$ and $b \geqslant 0$.

We take the boundary conditions to be

$$
\frac{\partial \theta}{\partial x}(0, t)=f(t), \quad \frac{\partial \theta}{\partial x}(1, t)=g(t), \quad u(0, t)=u(1, t)=0 \quad(t \geqslant 0) .
$$

We write $h=g-f$ for the net flow of heat: thus there is a net flow of heat into the slab at any instant $t$ at which $h(t)>0$, and there is a net flow of heat out of the slab at any instant at which $h(t)<0$. Since we are interested in drawing comparisons with the classical theory for a rigid conductor we have supposed the faces $x=0$ and $x=1$ to be clamped.

A preliminary lemma involves the function

$$
r(t)=a^{-1} \max _{0 \leqslant x, r \leqslant 1}\left|\frac{\partial \sigma}{\partial t}(x, t)-\frac{\partial \sigma}{\partial t}(y, t)\right|,
$$


whose values are finite if the stress-rate $\partial \sigma / \partial t$ is continuous in $S(0)$. This is certainly the case if, as we suppose, $\theta \in C^{2}$ and $u \in C^{3}$ in $S(0)$.

LemMA. Let $\theta \in C^{2}, u \in C^{3}$ in $S(0)$ and let $\theta, u$ satisfy the thermoelastic equations (1), (2) and the boundary conditions (3). Then $\theta$ has the maximum property in $S(0)$ if $h+r \leqslant 0$ in $[0, \infty)$ and $\theta$ has the minimum property in $S(0)$ if $h \geqslant r$ in $[0, \infty)$.

At first sight, the Lemma would appear to be of little value because we have no prior knowledge of $r$ in general. However, we shall find that it is possible to estimate $r$ in a satisfactory way. We do have prior knowledge of $r$ in the important quasi-static theory $(b=0)$ for then the stress $\sigma$ is independent of $x$, and $r$ vanishes identically. In that case we can immediately draw the conclusion:

Theorem. Suppose, in addition to the hypotheses of the Lemma, that $b=0$. Then $\theta$ has the maximum property in $S(0)$ if $h \leqslant 0$ in $[0, \infty)$, and $\theta$ has the minimum property in $S(0)$ if $h \geqslant 0$ in $[0, \infty)$.

The fact that $b$ is usually small by comparison with unity suggests that Theorem 1 is close to the truth even when inertia is taken into account. It will be noted that we have imposed no restriction upon the constant a beyond that it be positive.

The proof of the lemma is straightforward. We integrate Eq. (1) with respect to $x$ and we appeal to the boundary conditions (3) to find that

$$
\int_{0}^{1} \frac{\partial \theta}{\partial t} d x=g-f=h
$$

and we integrate the equation

$$
\partial u / \partial x=a \theta+\sigma
$$

and appeal to the boundary conditions again to find

$$
\int_{0}^{1} \sigma d x=-a \int_{0}^{1} \theta d x
$$

and, therefore,

$$
\int_{0}^{1} \frac{\partial \sigma}{\partial t} d x=-a h .
$$

Next, we differentiate (4) with respect to $t$ and substitute for $\partial^{2} u / \partial x \partial t$ in (1) and obtain the equations

$$
\begin{aligned}
\frac{\partial^{2} \theta}{\partial x^{2}} & =\left(1+a^{2}\right) \frac{\partial \theta}{\partial t}+a \frac{\partial \sigma}{\partial t} \\
& =\left(1+a^{2}\right) \frac{\partial \theta}{\partial t}-a^{2} h+a\left(\frac{\partial \sigma}{\partial t}-\int_{0}^{1} \frac{\partial \sigma}{\partial t}(z, t) d z\right)
\end{aligned}
$$

The first mean value theorem for integrals implies that there is $y \in[0,1]$, depending upon $t$, with

$$
\int_{0}^{1} \frac{\partial \sigma}{\partial t}(z, t) d z=\frac{\partial \sigma}{\partial t}(y, t)
$$


and, consequently, that we can estimate the third term on the right-hand side of (5) as

$$
\left|a\left(\frac{\partial \sigma}{\partial t}(x, t)-\int_{0}^{l} \frac{\partial \sigma}{\partial t}(z, t) d z\right)\right| \leqslant a\left|\frac{\partial \sigma}{\partial}(x, t)-\frac{\partial \sigma}{\partial t}(y, t)\right| \leqslant a^{2} r(t) .
$$

On returning to (5) we see that this estimate implies the inequality

$$
\frac{\partial^{2} \theta}{\partial x^{2}} \geqslant\left(1+a^{2}\right) \frac{\partial \theta}{\partial t}
$$

if $h+r \leqslant 0$ and, hence, that $\theta$ has the maximum property in $S(0)$. Similarly,

$$
\frac{\partial^{2} \theta}{\partial x^{2}} \leqslant\left(1+a^{2}\right) \frac{\partial \theta}{\partial t}
$$

if $h \geqslant r$ and then $\theta$ has the minimum property in $S(0)$.

In order to cope with the case in which $b$ is positive we introduce the second-order energy

$$
E=\frac{1}{2} \int_{0}^{1}\left(\left|\frac{\partial^{2} \theta}{\partial t^{2}}\right|^{2}+\left|\frac{\partial^{3} u}{\partial x \partial t^{2}}\right|^{2}+b\left|\frac{\partial^{3} u}{\partial t^{3}}\right|^{2}\right) d x
$$

We denote derivatives of $f, g, h$ by primes.

THEOREM 2. Suppose that $b>0$, that $\theta, u \in C^{4}$ in $S(0)$ and that $\theta, u$ satisfy the thermoelastic equations (1), (2) and the boundary conditions (3), where

(i) $|h(t)| \rightarrow \infty$ as $t \rightarrow+\infty$,

(ii) $h^{\prime}(t)=o(|h(t)|)$,

(iii) $\int_{0}^{t}\left(\left|f^{\prime \prime}(s)\right|^{2}+\left|g^{\prime \prime}(s)\right|^{2}\right) d s=o\left(|h(t)|^{2}\right)$.

Then there is a finite $t_{0} \geqslant 0$ such that $\theta$ has the maximum \{minimum property in $S\left(t_{0}\right)$ according as $h(t) \rightarrow-\infty\{+\infty\}$.

The hypotheses in $f, g, h$ are satisfied if, for example, $f$ and $g$ are polynomials

$$
f(t)=A_{0}+A_{1} t+\cdots+A_{n} t^{n}, \quad g(t)=B_{0}+B_{1} t+\cdots+B_{n} t^{n},
$$

of the same degree $n \geqslant 1$ and with $A_{n} \neq B_{n}$.

We might regard the hypothesis (iii) as saying that when we calculate the net heat flow $h=g-f$, by subtracting $f$ from $g$, the amount of cancellation is not excessive.

If one of the faces is insulated, that is if $f=0$ or $g=0$, (iii) reduces to the condition

(iv) $\int_{0}^{t}\left|h^{\prime \prime}(s)\right|^{2} d s=o\left(|h(t)|^{2}\right)$

on the net heat flow.

The same remark applies if $\theta$ is an even function with respect to the mid-plane $x=\frac{1}{2}$, in the sense that $\theta(x, t)=\theta(1-x, t)$ in $S(0)$, for then $-f=g=\frac{1}{2} h$.

The conditions (i), (ii), (iv) are satisfied if $h$ is any non-constant polynomial.

We begin the proof by observing that

$$
\frac{\partial \sigma}{\partial t}(z, t)-\frac{\partial \sigma}{\partial t}(y, t)=\int_{y}^{z} \frac{\partial^{2} \sigma}{\partial x \partial t} d x=b \int_{y}^{z} \frac{\partial^{3} u}{\partial t^{3}} d x
$$


and, therefore,

$$
r(t) \leqslant \frac{b}{a} \int_{0}^{1}\left|\frac{\partial^{3} u}{\partial t^{3}}\right| d x \leqslant \frac{b}{a} \sqrt{\int_{0}^{1}\left|\frac{\partial^{3} u}{\partial t^{3}}\right|^{2} d x} \leqslant \sqrt{\frac{2 b}{a^{2}} E(t)} .
$$

Thus, in order to estimate $r$ it is enough to estimate $E$, and that we can do with the aid of energy integral techniques.

If we set

$$
\begin{aligned}
& F=\frac{1}{2}\left(\left|\frac{\partial^{2} \theta}{\partial t^{2}}\right|^{2}+\left|\frac{\partial^{3} u}{\partial x \partial t^{2}}\right|^{2}+\left|\frac{\partial^{3} u}{\partial t^{3}}\right|^{2}\right) . \\
& G=\frac{\partial^{2} \theta}{\partial t^{2}} \cdot \frac{\partial^{3} \theta}{\partial x \partial t^{2}}+\frac{\partial^{3} u}{\partial t^{3}} \cdot \frac{\partial^{3} u}{\partial x \partial t^{2}}-a \frac{\partial^{2} \theta}{\partial t^{2}} \cdot \frac{\partial^{3} u}{\partial t^{3}}
\end{aligned}
$$

the thermoelastic equations imply that

$$
\frac{\partial G}{\partial x}-\frac{\partial F}{\partial t}=\left|\frac{\partial^{3} \theta}{\partial x \partial t^{2}}\right|^{2}
$$

Thus, if we apply Green's theorem

$$
\iint_{R}\left(\frac{\partial G}{\partial x}-\frac{\partial F}{\partial t}\right) d x d t=\int_{\partial R} F d x+G d t
$$

to any rectangle $R=[0,1] \times[0, T]$, where $\partial R$ is the (entire) boundary of $R$ oriented in the positive sense, we arrive at the equation

$$
\begin{aligned}
& \int_{0}^{T} \int_{0}^{1}\left|\frac{\partial^{3} \theta}{\partial x \partial t^{2}}\right|^{2} d x d t+E(T) \\
& \quad=E(0)-\int_{0}^{T} \frac{\partial^{2} \theta}{\partial t^{2}}(0, t) f^{\prime \prime}(t) d t+\int_{0}^{T} \frac{\partial^{2} \theta}{\partial t^{2}}(1, t) g^{\prime \prime}(t) d t
\end{aligned}
$$

Next, we recall that

$$
\int_{0}^{1} \frac{\partial \sigma}{\partial t} d x=h
$$

and, therefore,

$$
\int_{0}^{1} \frac{\partial^{2} \theta}{\partial t^{2}} d x=h^{\prime}
$$

Accordingly, if we integrate the identities

$$
\begin{aligned}
\frac{\partial}{\partial x}\left((1-x) \frac{\partial^{2} \theta}{\partial t^{2}}\right) & =-\frac{\partial^{2} \theta}{\partial t^{2}}+(1-x) \frac{\partial^{3} \theta}{\partial x \partial t^{2}} \\
\frac{\partial}{\partial x}\left(x \frac{\partial^{2} \theta}{\partial t^{2}}\right) & =\frac{\partial^{2} \theta}{\partial t^{2}}+x \frac{\partial^{3} \theta}{\partial x \partial t^{2}}
\end{aligned}
$$


we obtain the formulae

$$
\begin{aligned}
-\frac{\partial^{2} \theta}{\partial t^{2}}(0, t) & =-h^{\prime}+\int_{0}^{1}(1-x) \frac{\partial^{3} \theta}{\partial x \partial t^{2}} d x, \\
\frac{\partial^{2} \theta}{\partial t^{2}}(1, t) & =h^{\prime}+\int_{0}^{1} x \frac{\partial^{3} \theta}{\partial x \partial t^{2}} d x,
\end{aligned}
$$

and, when we substitute into the right-hand side of (6), we see that

$$
\begin{aligned}
\int_{0}^{T} \int_{0}^{1}\left|\frac{\partial^{3} \theta}{\partial x \partial t^{2}}\right|^{2} d x d t+E(T) \\
=E(0)+\int_{0}^{T} \int_{0}^{1} f^{\prime \prime}(t)(1-x) \cdot \frac{\partial^{3} \theta}{\partial x \partial t^{2}} d x d t \\
\quad+\int_{0}^{T} \int_{0}^{1} g^{\prime \prime}(t) x \cdot \frac{\partial^{3} \theta}{\partial x \partial t^{2}} d x d t+\int_{0}^{T} h^{\prime}(t) h^{\prime \prime}(t) d t
\end{aligned}
$$

Since the arithmetic-geometric mean inequality leads to the estimates

$$
\begin{aligned}
& \int_{0}^{T} \int_{0}^{1} f^{\prime \prime}(t)(1-x) \frac{\partial^{3} \theta}{\partial x \partial t^{2}} d x d t+\int_{0}^{T} \int_{0}^{1} g^{\prime \prime}(t) x \frac{\partial^{3} \theta}{\partial x \partial t^{2}} d x d t \\
& \leqslant \frac{1}{2} \int_{0}^{T} \int_{0}^{1}\left(\left|f^{\prime \prime}(t)\right|^{2}(1-x)^{2}+\left|\frac{\partial^{3} \theta}{\partial x \partial t^{2}}\right|^{2}\right) d x d t \\
&+\frac{1}{2} \int_{0}^{T} \int_{0}^{1}\left(\left|g^{\prime \prime}(t)\right|^{2} x^{2}+\left|\frac{\partial^{3} \theta}{\partial x \partial t^{2}}\right|^{2}\right) d x d t \\
&= \frac{1}{6} \int_{0}^{T}\left(\left|f^{\prime \prime}(t)\right|^{2}+\left|g^{\prime \prime}(t)\right|^{2}\right) d t+\int_{0}^{T} \int_{0}^{1}\left|\frac{\partial^{3} \theta}{\partial x \partial t^{2}}\right|^{2} d x d t,
\end{aligned}
$$

we conclude that

$$
E(T) \leqslant E(0)-\frac{1}{2} h^{\prime}(0)^{2}+\frac{1}{6} \int_{0}^{T}\left(\left|f^{\prime \prime}(t)\right|^{2}+\left|g^{\prime \prime}(t)\right|^{2}\right) d t+\frac{1}{2} h^{\prime}(T)^{2}
$$

and, in the light of the hypotheses (i), (ii), (iii), that $E(t)=o\left(|h(t)|^{2}\right.$ ) as $t \rightarrow+\infty$. Thus we can choose $t_{0} \geqslant 0$ in such a way that $2 b E \leqslant a^{2} h^{2}$ in the interval $\left[t_{0}, \infty\right)$ and, without any loss in generality, we may suppose that $h$ is of constant $\operatorname{sign}$ in $\left[t_{0}, \infty\right)$. It follows that $r \leqslant|h|$ in $\left[t_{0}, \infty\right)$ and, moreover, that $h+r \leqslant 0$ in $\left[t_{0}, \infty\right)$ if $h(t) \rightarrow-\infty$ and that $h \geqslant r$ in $\left[t_{0}, \infty\right)$ if $h(t) \rightarrow+\infty$. In the former case, the Lemma implies that $\theta$ has the maximum property in $S\left(t_{0}\right)$ and, in the latter, that $\theta$ has the minimum property in $S\left(t_{0}\right)$, which completes the

2. A comparison theorem. We use the results of the preceding section to compare the temperature predicated by solving the thermoelastic equations with that predicated by solving a heat equation.

TheOrem 3. Let $\theta, u \in C^{4}$ in $S(0)$, let $\theta, u$ satisfy the thermoelastic equations (1), (2) and the boundary condition

$$
u(0, t)=u(1, t)=0 \quad(t \geqslant 0)
$$


and suppose that

$$
f(t)=\frac{\partial \theta}{\partial x}(0, t), \quad g(t)=\frac{\partial \theta}{\partial x}(1, t), \quad h=g-f,
$$

satisfy the hypotheses (i), (ii), (iii).

Let $\varphi \in C^{2}$ in $S(0)$, let $\varphi$ be a solution of the heat equation

$$
\frac{\partial^{2} \varphi}{\partial x^{2}}=\left(1+a^{2}\right) \frac{\partial \varphi}{\partial t}
$$

and let

$$
\varphi(0, t)=\theta(0, t), \quad \varphi(1, t)=\theta(1, t) \quad(t \geqslant 0) .
$$

Then there is a finite $t_{0} \geqslant 0$ such that $\theta \geqslant \varphi$ or $\theta \leqslant \varphi$ throughout $S\left(t_{0}\right)$, according as $h(t) \rightarrow+\infty$ or $h(t) \rightarrow-\infty$.

Once again the hypothesis (iii) can be replaced by the hypothesis (iv) on $h$ alone when one face is insulated, or when $\theta$ is even with respect to the mid-plane.

The Theorem compares $\theta$ with a $\varphi$ which takes the same values on the faces $x=0$ and $x=1$ as does $\theta$, and which is a solution of the heat equation in which the (scaled) diffusivity 1 is replaced by $\left(1+a^{2}\right)^{-1}$. No restriction is placed upon the values taken by $\theta, u, \partial u / \partial t$, or $\varphi$ on the initial line segment $[0,1] \times\{0\}$, and $\theta$ and $\varphi$ need not coincide there. It is supposed that information is available about the behaviour of the heat flow across the faces $x=0$ and $x=1$ in the thermoelastic problem.

In order to prove the Theorem we use the elementary remark that: if $p \in C^{1}$ in $[0,1]$ and $p(0)=p(1)=0$, the quotient $p(x) / x(1-x)$ is bounded in the open interval $(0,1)$. We also need explicit, though not best possible, bounds on the quotient when $p(x)=\sin \pi x$. These are provided by the inequalities

$$
2 x(1-x) \leqslant \sin \pi x \leqslant 2 \pi x(1-x),
$$

which hold in $[0,1]$. It is enough to verify them in $\left[0, \frac{1}{2}\right]$.

In that interval

$$
2 x \leqslant \sin \pi x \leqslant \pi x, \quad \frac{1}{2} \leqslant 1-x \leqslant 1,
$$

the first two bounds being valid because $\sin \pi x$ is a concave function of $x$, and the required inequalities follow.

We may suppose $h(t) \rightarrow+\infty$ as $t \rightarrow+\infty$, for the alternative case is covered by arguing with $-\theta$ in place of $\theta$ and $-\varphi$ in place of $\varphi$.

If we return to equation (5), which we derived in the course of proving the Lemma, we see that $\theta$ satisfies the inequality

$$
\frac{\partial^{2} \theta}{\partial x^{2}} \leqslant\left(1+a^{2}\right) \frac{\partial \theta}{\partial t}-a^{2} h+a^{2} r,
$$

where, as we know,

$$
0 \leqslant r \leqslant \sqrt{\frac{2 b}{a^{2}} E}=o(h) .
$$


Thus there is $t_{1} \geqslant 0$ such that $r(t) \leqslant \frac{1}{2} h(t)$ and $h(t) \geqslant 1$ when $t \geqslant t_{1}$. Consider

$$
\begin{aligned}
\psi(x, t)= & \theta(x, t)-\varphi(x, t)-\frac{1}{4} a^{2} x(1-x) \\
& +A \sin \pi x \cdot \exp \left(-\frac{\pi^{2}}{1+a^{2}}\left(t-t_{1}\right)\right),
\end{aligned}
$$

in which the constant $A>0$ will be chosen later. We have

$$
\begin{aligned}
\frac{\partial^{2} \psi}{\partial x^{2}}-\left(1+a^{2}\right) \frac{\partial \psi}{\partial t} & =\frac{\partial^{2} \theta}{\partial x^{2}}-\left(1+a^{2}\right) \frac{\partial \theta}{\partial t}+\frac{1}{2} a^{2} \\
& \leqslant-a^{2} h+a^{2} r+\frac{1}{2} a^{2} \leqslant-\frac{1}{2} a^{2} h+\frac{1}{2} a^{2} \leqslant 0
\end{aligned}
$$

in the half-strip $S\left(t_{1}\right)$. Since $\varphi$ coincides with $\theta$ on the vertical sides of $S(t), \psi$ vanishes on the vertical sides. On the base

$$
\begin{aligned}
\psi\left(x, t_{1}\right) & =\theta\left(x, t_{1}\right)-\varphi\left(x, t_{1}\right)-\frac{1}{4} a^{2} x(1-x)+A \sin \pi x \\
& \geqslant x(1-x)\left(B-\frac{1}{4} a^{2}+2 A\right),
\end{aligned}
$$

where $B$ is a lower bound on the quotient

$$
\frac{\theta\left(x, t_{1}\right)-\varphi\left(x, t_{1}\right)}{x(1-x)},
$$

and, therefore, we can arrange that $\psi \geqslant 0$ on the base of $S\left(t_{1}\right)$ by choosing $A=$ $\max \left\{0, \frac{1}{8} a^{2}-\frac{1}{2} B\right\}$. With $A$ chosen in this way, we have $\psi \geqslant 0$ throughout $S\left(t_{1}\right)$ and, therefore,

$$
\begin{aligned}
\theta-\varphi & \geqslant \frac{1}{4} a^{2} x(1-x)-A \sin \pi x \cdot \exp \left(-\frac{\pi^{2}}{1+a^{2}}\left(t-t_{1}\right)\right) \\
& \geqslant x(1-x)\left(\frac{1}{4} a^{2}-2 \pi A \cdot \exp \left(-\frac{\pi^{2}}{1+a^{2}}\left(t-t_{1}\right)\right)\right) .
\end{aligned}
$$

Thus if we take $t_{0}>t_{1}$ sufficiently large we shall have $\theta \geqslant \varphi$ throughout the half-strip $S\left(t_{0}\right)$, which proves the Theorem.

It will be noted that our proof depends upon equation (5), which depends in turn upon the hypothesis that the faces are clamped. Thus although the Theorem is an assertion about the temperature its validity depends in part upon the boundary conditions on the displacement; this is just what we should expect from a coupled theory.

\section{REFERENCES}

[1] D. E. Carlson, Linear thermoelasticity, Encyclopedia of Physics, Vol. VIa/2, Springer-Verlag, Berlin, 1972

[2] W. A. Day, A comment on approximations to the temperature in dynamic linear thermoelasticity, Arch. Rational Mech. Anal. 85, 237-250 (1984)

[3] W. A. Day, A property of the heat equation which extends to the thermoelastic equations, Arch. Rational Mech. Anal. 83, 99-113 (1983)

[4] W. A. Day, Further remarks on a property of the equations of dynamic thermoelasticity, Arch. Rational Mech. Anal. 84, 69-81 (1983) 